\title{
Hemosuccus pancreaticus caused by in situ carcinoma of the pancreas
}

\section{A-77-year-old-woman was referred to our} hospital because of dilatation of the main duct in the pancreatic body, as seen on transabdominal ultrasonography. The patient had experienced several episodes of acute pancreatitis in the previous 2 years. Laboratory tests showed mild anemia (hemoglobin $11.7 \mathrm{~g} / \mathrm{dL}$; reference range: $12-16 \mathrm{~g} / \mathrm{dL}$ ). Levels of serum amylase, carcinoembryonic antigen, and carbohydrate antigen 19-9 were within normal limits. Computed tomography and magnetic resonance cholangiopancreatography revealed mild dilatation of the main pancreatic duct and demonstrated lack of a mass lesion. Endoscopic retrograde cholangiopancreatography (ERCP) showed stricture of the main duct in the head of the pancreas ( Fig. 1). Cytological analysis of the pancreatic juice showed no malignancy. During ERCP, a side-viewing upper endoscope showed blood oozing from the major papilla ( Fig. 2). Bleeding was intermittent and stopped after ERCP. Endoscopic ultrasonography (EUS) showed a small vague hypoechoic area adjacent to the stenotic duct of the pancreas.

These findings led to a clinical diagnosis of pancreatic cancer at an early stage, and pancreatic duodenectomy was performed. Histopathological examination showed intraductally spreading carcinoma in the main and branch duct of the pancreatic body, and inflammation and fibrosis adjacent to the pancreatic duct; a hemorrhage site was seen in the concomitant pancreatitis area ( Fig.3). The histopathological diagnosis was in situ carcinoma of the pancreas.

Hemosuccus pancreaticus is a rare cause of gastrointestinal bleeding. The bleeding is usually caused by rupture of a visceral artery aneurysm in chronic pancreatitis [1].

Although the cause of hemosuccus pancreaticus is mainly acute or chronic pancreatitis, an early stage pancreatic tumor may be a possible cause of hemosuccus pancreaticus, and such clues may lead to the diagnosis of pancreatic cancer at an early stage. Hemosuccus pancreaticus caused by a pancreatic tumor is extremely rare. To date, there have only been two reported cases in the English literature [2, 3]. In this case, we suspected that the small vessel wall failure due to concomitant pancreatitis of in situ carcinoma was the cause of hemosuccus pancreaticus. In situ carcinoma of the pancreas is regarded as an early stage of pancreatic cancer, with a better prognosis. The diagnosis of in situ carcinoma of the pancreas poses a great challenge to the clinician. The reasons for delayed diagnosis may be related to absence of early symptoms and lack of sufficient direct or indirect evidence of tumor.

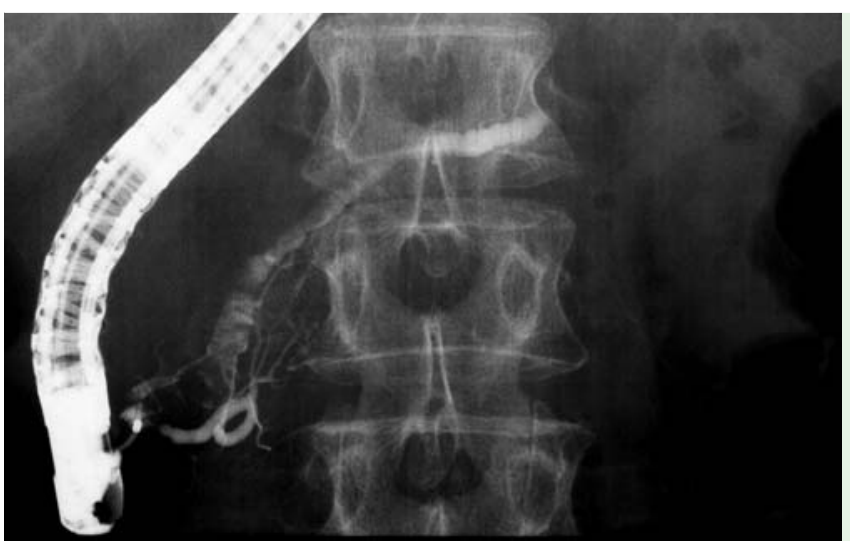

Fig. 1 Endoscopic retrograde cholangiopancreatography (ERCP) showed stricture of the main duct in the head of the pancreas.
Endoscopy_UCTN_Code_CCL_1AZ_2AB

Competing interests: None

\section{H. Inoue', M. Katurahara², Y. Hamada², K. Ninomiya', S. Tano², R. Takayama', K. Nojiri ${ }^{1}$, M. Tameda ${ }^{1}$, N. Horiki ${ }^{2}$, Y. Takei ${ }^{1}$}

${ }^{1}$ Department of Gastroenterology and Hepatology, Mie University Graduate School of Medicine, Mie, Japan

${ }^{2}$ Department of Endoscopy, Mie University Graduate School of Medicine, Mie, Japan

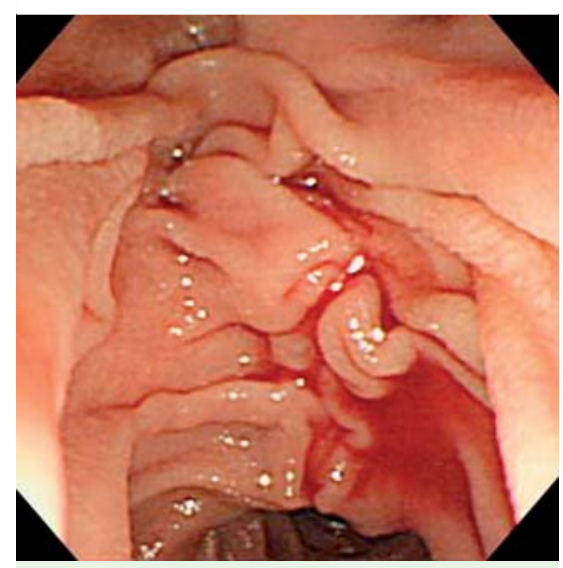

Fig. 2 Blood was detected oozing from the major papilla, by a side-viewing upper endoscope. 


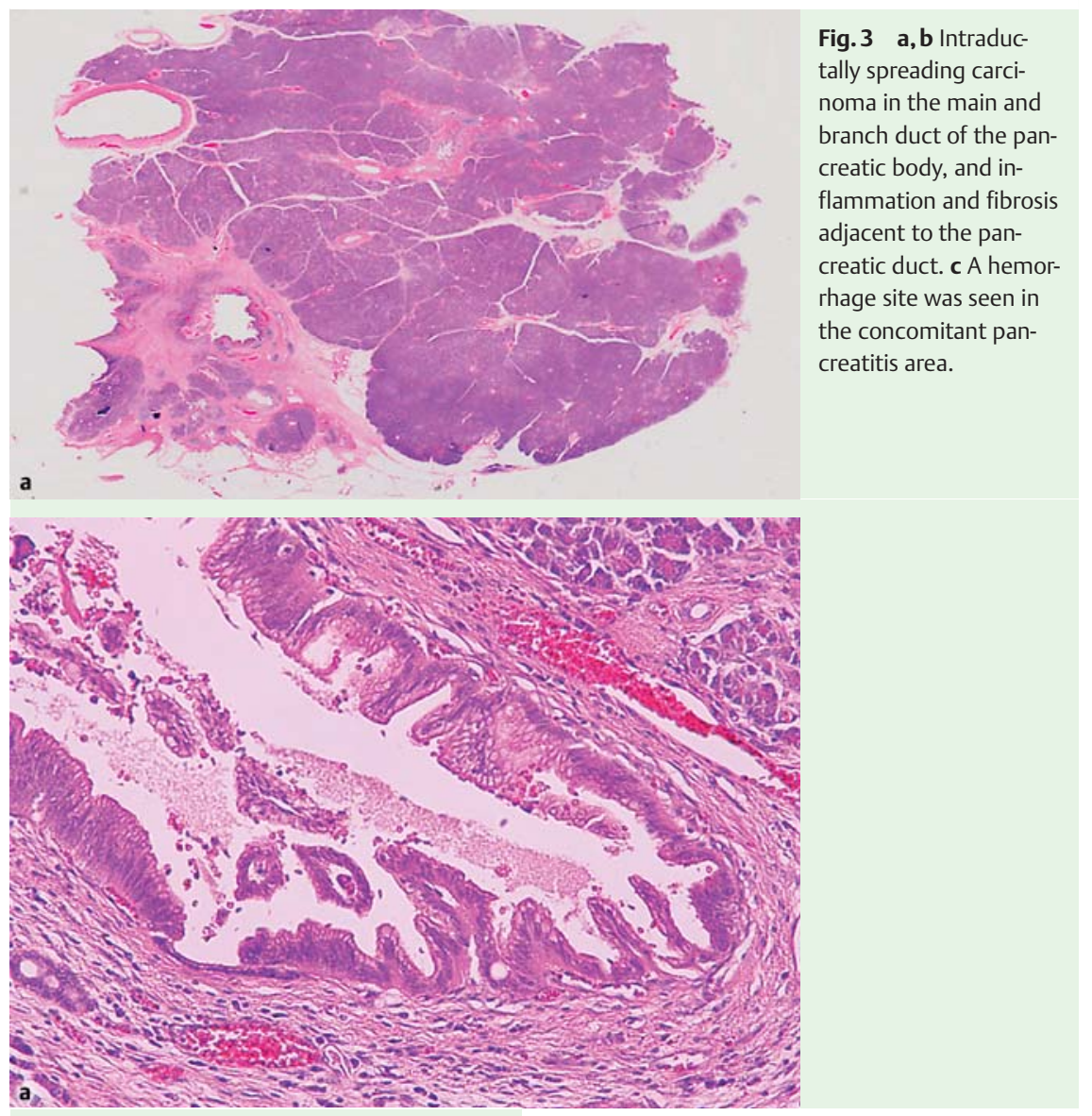

\section{References}

1 Kapoor S, Rao P, Sujoy $P$ et al. Hemosuccus pancreaticus: an uncommon cause of gastrointestinal hemorrhage: a case report. JOP 2004; 5: 373-376

2 Kurland J, Matthews T, Hoff E et al. Hemosuccus pancreaticus caused by metastatic renal cell carcinoma. Gastrointest Endosc 2007; 66: $1241-1242$

3 Shinzeki M, Hori Y, Fujino $Y$ et al. Mucinous cystic neoplasm of the pancreas presenting with hemosuccus pancreaticus: report of a case. Surg Today 2010; 40: 470-473

\section{Bibliography}

DOI http://dx.doi.org/

10.1055/s-0032-1309863

Endoscopy 2012; 44: E336-E337

(c) Georg Thieme Verlag KG

Stuttgart · New York

ISSN 0013-726X

\section{Corresponding author \\ H. Inoue, MD, PhD}

Department of Gastroenterology and Hepatology Mie University Graduate School of Medicine

2-174 Edobashi Tsu

Mie

Japan

Fax: +81-059-2325223

hiro1024@clin.medic.mie-u.ac.jp

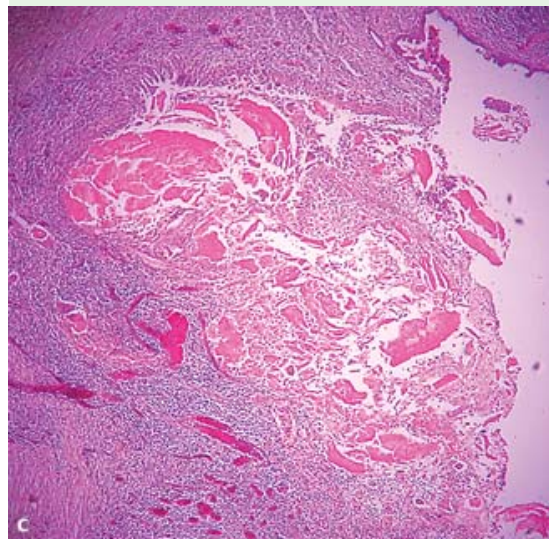

